\title{
Observer-based speed estimation method for sensorless vector control of induction motors
}

\author{
C.-M. Lee \\ C.-L.Chen
}

Indexing terms: Induction motors, Speed estimation, Vector control

\begin{abstract}
The method is based on an adaptive flux observer in the rotor-speed reference frame, in which a second-order Kalman filter is employed to modify the estimated rotor flux to improve the performance of speed estimation. The Kalman filter modifies the estimated rotor flux based on the measured stator currents. The estimated speed is used in the speed feedback for vector control and in the co-ordinate transformation for current controller. The proposed method has the advantage of saving much computation time in comparison with the reduced-order extended Kalman filter. Compared with the conventional adaptive observer, the proposed method has the advantage of better accuracy to follow the speed command under heavy loads. Experimental results show the effectiveness of the proposed method.
\end{abstract}

\section{Introduction}

Vector-controlled induction motor drives have been widely used in high-performance applications. Conventional vector control methods [1-6] require motor speed as a feedback signal. To obtain the speed information, transducers such as shaft-mounted tachogenerators, resolvers, or digital shaft position encoders are used, which degrade the system's reliability, especially in hostile environments.

Sensorless vector control is proposed to cope with the speed-sensing problem. Various approaches of sensorless vector control have been presented in the literature. H. Kubota et al. [7] proposed an adaptive flux observer to estimate the rotor flux and speed. The computation process of estimating the rotor speed is simple, and the estimated rotor flux is used to calculate the vector rotation angle. However, experimental results show that large speed errors appear under heavy loads.

Kalman filtering is known for obtaining high-accuracy estimates of state variables under noisy environments. Y.R. Kim et al. [8] used a fifth-order extended

\section{(C) IEE, 1998}

IEE Proceedings online no. 3251

Paper first received lst September 1997 and in revised form 27th January 1998

The authors are with the Power Electronics Laboratory, Department of Electrical Engineering, National Taiwan University, Taipei, Taiwan
Kalman filter to identify the speed of an induction motor. The performance of the speed estimation is excellent but the computation process consumes a lot of time. Y.S. Kim et al. [9] employed a reduced thirdorder extended Kalman filter to estimate the rotor speed. According to $[10,11]$, the number of multiplications in the Kalman filter is proportional to the third power of the state size. Therefore the reduced-order extended Kalman filter saves much computation time in comparison with the fifth-order extended Kalman filter, but is still time-consuming compared with the adaptive flux observer.

We propose a new speed estimation method which is based on an adaptive flux observer in the rotor speed reference frame. A second-order Kalman filter is then employed to modify the estimated rotor flux. The modified estimated rotor flux is substituted into the flux observer to improve the performance of the rotor speed estimation. The estimated speed takes place of the feedback signal for conventional vector control, and thus a sensorless vector control system is established. The proposed method saves much computation time compared with the reduced-order extended Kalman filter and has better performance under heavy loads than the conventional adaptive observer.

\section{Principles of proposed approach}

This Section is divided into five subsections: part A introduces the basic induction motor model, part B describes the flux observer developed by Kubota $e t$ al. [7], part $\mathrm{C}$ examines the details of implementing the second-order Kalman filter to estimate the rotor flux. The estimated rotor flux is to be substituted into the rotor-speed estimation equation developed in part $\mathrm{B}$ to improve the performance of speed estimation. Part D describes the process of the proposed method by a flow chart.

\subsection{Motor model}

The state equations of an induction motor in the rotorspeed reference frame can be expressed as follows:

$$
\begin{gathered}
\frac{d X(t)}{d t}=A \cdot X(t)+B \cdot U(t) \\
Y(t)=C \cdot X(t)
\end{gathered}
$$

where

$$
\begin{aligned}
& X=\left[\begin{array}{llll}
i_{d s} & i_{q s} & \phi_{d r} & \phi_{q r}
\end{array}\right]^{T} \\
& Y=\left[\begin{array}{ll}
i_{d s} & i_{q s}
\end{array}\right]^{T}
\end{aligned}
$$




$$
\begin{aligned}
& U=\left[\begin{array}{ll}
v_{d s} & v_{q s}
\end{array}\right]^{T} \\
& A= \\
& {\left[\begin{array}{cccc}
-\left(\frac{R_{s}}{\sigma L_{s}}+\frac{1-\sigma}{\sigma \tau_{r}}\right) & \omega_{r} & \frac{L_{m}}{\sigma L_{s} L_{r}} \frac{1}{\tau_{r}} & \omega_{r} \frac{L_{m}}{\sigma L_{s} L_{r}} \\
-\omega_{r} & -\left(\frac{R_{s}}{\sigma L_{s}}+\frac{1-\sigma}{\sigma \tau_{r}}\right) & -\omega_{r} \frac{L_{m}}{\sigma L_{s} L_{r}} & \frac{L_{m}}{\sigma L_{s} L_{r}} \frac{1}{\tau_{r}} \\
\frac{L_{m}}{\tau_{r}} & 0 & -\frac{1}{\tau_{r}} & 0 \\
0 & \frac{L_{m}}{\tau_{r}} & 0 & -\frac{1}{\tau_{r}}
\end{array}\right]} \\
& B=\left[\begin{array}{cc}
\frac{1}{\sigma L_{s}} & 0 \\
0 & \frac{1}{\sigma L_{s}} \\
0 & 0 \\
0 & 0
\end{array}\right] \quad C=\left[\begin{array}{cccc}
1 & 0 & 0 & 0 \\
0 & 1 & 0 & 0
\end{array}\right] \\
& i_{s}=\left[\begin{array}{lll}
i_{d s} & i_{q s}
\end{array}\right]^{T} \text { stator current } \\
& \phi_{r}=\left[\begin{array}{ll}
\phi_{d r} & \phi_{q r}
\end{array}\right]^{T} \text { rotor flux } \\
& v_{s}=\left[\begin{array}{ll}
v_{d s} & v_{q s}
\end{array}\right]^{T} \text { stator voltage }
\end{aligned}
$$

\subsection{Adaptive flux observer}

The state observer in the rotor-speed reference frame is written as follows [7]:

$$
\frac{d}{d t} \hat{X}=\hat{A} \hat{X}+B U+G\left(\hat{i}_{s}-i_{s}\right)
$$

where ^signifies the estimated quantities and $G$ is the observer gain matrix which is to be determined so that eqn. 3 can be stable. Note that all the state variables are in the rotor-speed reference frame. The estimation error of the states can be written as

$$
\frac{d}{d t} e=(A+G C) e-\Delta A \hat{X}
$$

where

$$
\begin{aligned}
& e=X-\hat{X} \\
& \Delta A=\hat{A}-A \\
& =\left[\begin{array}{cccc}
0 & \Delta \omega_{r} & 0 & \Delta \omega_{r} / k \\
-\Delta \omega_{r} & 0 & -\Delta \omega_{r} / k & 0 \\
0 & 0 & 0 & 0 \\
0 & 0 & 0 & 0
\end{array}\right] \\
& k=\left(\sigma L_{s} L_{r}\right) / L_{m}, \quad \Delta \omega_{r}=\hat{\omega}_{r}-\omega_{r}
\end{aligned}
$$

Define the following Lyapunov function candidate [7]:

$$
V=e^{T} e+\left(\hat{\omega}_{r}-\omega_{r}\right)^{2} / \lambda
$$

where $\lambda$ is a positive constant. The time derivative of $V$ is

$$
\begin{aligned}
\frac{d}{d t} V= & e^{T}\left\{(A+G C)^{T}+(A+G C)\right\} e \\
& -2 \Delta \omega_{r}\left(e_{i d s}\left(\hat{i}_{q s}+\hat{\phi}_{q T} / k\right)\right. \\
& \left.\quad-e_{i q s}\left(\hat{i}_{d s}+\hat{\phi}_{d r} / k\right)\right) \\
& +2 \Delta \omega_{r} \frac{d}{d t} \hat{\omega}_{r} / \lambda
\end{aligned}
$$

where $e_{i d s}=i_{d s}-\hat{i}_{d s}, e_{i q s}=i_{q s}-\hat{i}_{q s}$. By equating the second and the third term of eqn. 6 , we obtain the following speed estimation equation:

$$
\begin{aligned}
& \frac{d}{d t} \hat{\omega}_{r}=\frac{\lambda}{k}\left[e_{i d s}\left(k \hat{i}_{q s}+\hat{\phi}_{q r}\right)-e_{i q s}\left(k \hat{i}_{d s}+\hat{\phi}_{d r}\right)\right]_{(7)} \\
& \hat{\omega}_{r}=K_{p} \int\left[e_{i d s}\left(k \hat{i}_{q s}+\hat{\phi}_{q r}\right)-e_{i q s}\left(k \hat{i}_{d s}+\hat{\phi}_{d r}\right)\right] d t
\end{aligned}
$$

where $K_{p}$ is a proportional constant. If the observer gain matrix $G$ is chosen so that the first term of eqn. 6 is negative-semidefinite, the adaptive flux observer will be stable according to the Lyapunov's direct method. For ease of implementation, we choose $G=0$. Then the first term of eqn. 6 will be negative-semidefinite since an induction motor itself is stable.

\subsection{Second-order Kalman filter}

The estimated rotor flux $\hat{\phi}_{d r}$, $\hat{\phi}_{q r}$ used in eqn. 8 is obtained by using the state observer (eqn. 3) and assuming that the state variables $X(t)$ and input variables $U(t)$ are constant during a discrete step period. These estimated values may suffer large errors if the state variables change rapidly. For example, the measured stator currents (sinusoidal) can change rapidly under heavy-load conditions, especially in high-speed commands. Therefore we implement a second-order Kalman filter to modify the estimated rotor flux in eqn. 8, to improve the accuracy of the rotor-speed estimation. For the purpose of applying Kalman filtering techniques, a state model and an output model must be derived. The details are as follows. Rewrite eqn. 1 in discrete form

$$
\begin{aligned}
& {\left[\begin{array}{c}
i_{d s}(k+1) \\
i_{q s}(k+1) \\
\phi_{d r}(k+1) \\
\phi_{q r}(k+1)
\end{array}\right]=} \\
& {\left[\begin{array}{cc}
1-\left(\frac{R_{s}}{\sigma L_{s}}+\frac{1-\sigma}{\sigma \tau_{r}}\right) \Delta t & \omega_{r} \Delta t \\
-\omega_{r} \Delta t & 1-\left(\frac{R_{s}}{\sigma L_{s}}+\frac{1-\sigma}{\sigma \tau_{r}}\right) \Delta t \\
\frac{L_{m}}{\tau_{r}} \Delta t & 0 \\
0 & \frac{L_{m}}{\tau_{r}} \Delta t
\end{array}\right.} \\
& \frac{L_{m}}{\sigma L_{s} L_{r}} \frac{1}{\tau_{r}} \Delta t \quad \omega_{r} \frac{L_{m}}{\sigma L_{s} L_{r}} \Delta t \\
& -\omega_{r} \frac{L_{m}}{\sigma L_{s} L_{r}} \Delta t \quad \frac{L_{m}}{\sigma L_{s} L_{r}} \frac{1}{\tau_{r}} \Delta t \\
& 1-\frac{1}{\tau_{r}} \Delta t \quad 0 \\
& 0 \quad 1-\frac{1}{\tau_{r}} \Delta t \\
& \times\left[\begin{array}{c}
i_{d s}(k) \\
i_{q s}(k) \\
\phi_{d r}(k) \\
\phi_{q r}(k)
\end{array}\right]+\left[\begin{array}{cc}
\frac{1}{\sigma L_{s}} \Delta t & 0 \\
0 & \frac{1}{\sigma \bar{L}_{s}} \Delta t \\
0 & 0 \\
0 & 0
\end{array}\right]\left[\begin{array}{c}
v_{d s} \\
v_{q s}
\end{array}\right]
\end{aligned}
$$

Define the discrete state-model and output model as follows:

state model (lower two rows of eqn. 9):

$$
\begin{aligned}
{\left[\begin{array}{c}
\phi_{d r}(k+1) \\
\phi_{q r}(k+1)
\end{array}\right]=} & {\left[\begin{array}{cc}
1-\frac{1}{\tau_{r}} \Delta t & 0 \\
0 & 1-\frac{1}{\tau_{r}} \Delta t
\end{array}\right]\left[\begin{array}{l}
\phi_{d r}(k) \\
\phi_{q r}(k)
\end{array}\right] } \\
& +\left[\begin{array}{c}
\frac{L_{\eta_{n}}}{\tau_{r}} \Delta t \cdot i_{d s}(k) \\
\frac{L_{m n}}{\tau_{r}} \Delta t \cdot i_{q s}(k)
\end{array}\right]
\end{aligned}
$$

output model (upper two rows of eqn. 9): 


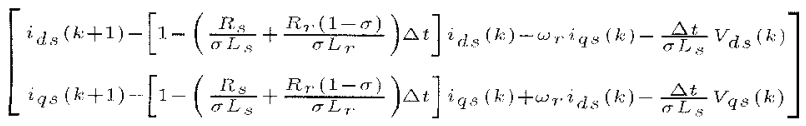

$$
\begin{aligned}
& =\left[\begin{array}{cc}
\frac{L_{m}}{\sigma L_{s} L_{r}} \frac{1}{\tau_{r}} \Delta t & \omega_{r} \frac{L_{m}}{\sigma L_{s} L_{r}} \Delta t \\
-\omega_{r} \frac{L_{m_{r}}}{\sigma L_{r} L_{r}} \Delta t & \frac{L_{m}}{\sigma L_{s} L_{r}} \frac{1}{\tau_{r}} \Delta t
\end{array}\right]\left[\begin{array}{c}
\phi_{d r}(k) \\
\phi_{q r}(k)
\end{array}\right]
\end{aligned}
$$

Consider this discrete-state model and output model with noises

$$
\begin{gathered}
x(k+1)=F(k) x(k)+u(k)+w(k) \\
z(k)=H(k) x(k)+v(k)
\end{gathered}
$$

where

$$
\begin{aligned}
& x(k)=\left[\begin{array}{ll}
\phi_{d r}(k) & \phi_{q r}(k)
\end{array}\right] \\
& z(k+1)=
\end{aligned}
$$

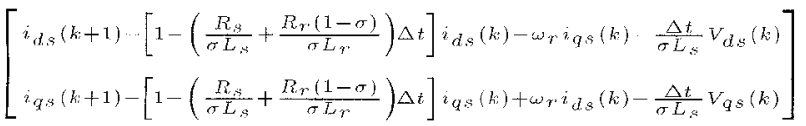

$w(k) \quad=$ random noise matrix of state model

$v(k)=$ random noise matrix of output model

$E\left[w_{i}\right] \quad=0$

$E\left[v_{i}\right] \quad=0$

$E\left[w w^{T}\right]=0$

$E\left[w_{i} w_{j}^{T}\right]=0 \quad i \neq j$

$$
=q \delta_{i i} \quad i=j
$$

$E\left[v v^{T}\right]=R$

$E\left[v_{i} v_{j}^{T}\right]=0 \quad i \neq j$

$$
=r \delta_{i i} \quad i=j
$$

$E\left[w_{i} v_{i}{ }^{T}\right]=0$

$E[x(0)]=\tilde{x}(0)$

$E\left[(x(0)-\tilde{x}(0))(x(0)-\tilde{x}(0))^{T}\right]=P(0)$

$P(0)=$ estimation error covariance matrix at time $t=0$

$\delta_{i i} \quad=$ kronecker delta

Note that in the definition of the output model eqn. 13, the stator currents are actually measured values [9], so $i_{d s}(k+1)$ and $i_{q s}(k+1)$ represent the measured stator current at the present state, and $i_{d s}(k)$ and $i_{q s}(k)$ represent the measured stator current at the previous state. Thus, we can apply the Kalman filtering technique to estimate the rotor flux by the following steps [8]:

(i) Prediction of state:

$$
\hat{x}(k+1 \mid k)=F(k) \hat{x}(k \mid k)+u(k)
$$

(ii) Estimation of error covariance matrix:

$$
P(k+1 \mid k)=F(k) P(k \mid k) F^{T}(k)+Q(k)
$$

(iii) Computation of Kalman filter gain:

$$
\begin{aligned}
K(k+1)= & P(k+1 \mid k) H^{T}(k+1) \\
& \times\left[H(k+1) P(k+1 \mid k) H^{T}(k+1)\right. \\
& +R(k+1)]^{-1}
\end{aligned}
$$

(iv) Update of the error covariance matrix:

$$
P(k+1 \mid k+1)=[I-K(k+1) H(k+1)] P(k+1 \mid k)
$$

(v) State estimation:

$$
\begin{array}{r}
\hat{x}(k+1 \mid k+1)=\hat{x}(k+1 \mid k)+K(k+1)[z(k+1) \\
-H(k+1) \hat{x}(k+1 \mid k)]
\end{array}
$$

The modified estimated rotor flux obtained utilising the second-order Kalman filter is then substituted into the speed estimation equation (eqn. 8) to obtain better estimation of the rotor speed. Since there is no change in eqn. 8 except the improved flux estimation, the stability condition stated in the previous Section would not deteriorate.

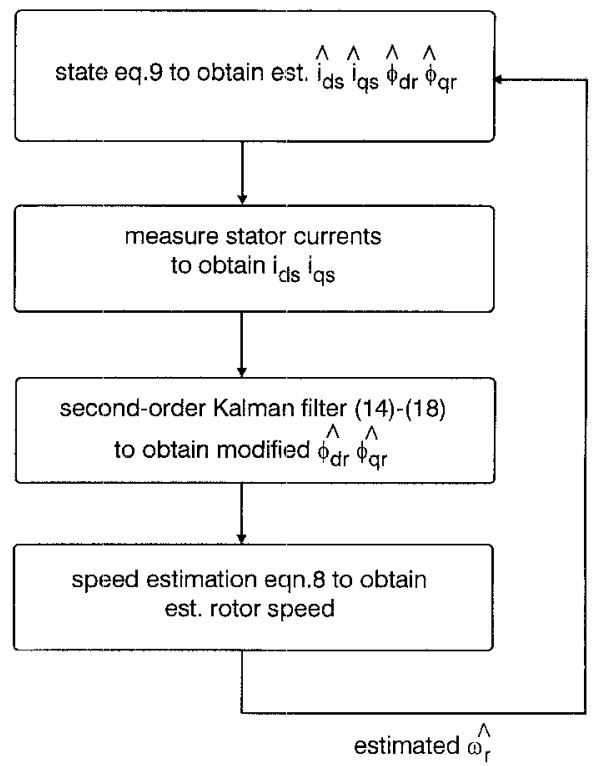

Fig. 1 Flowchart of proposed speed estimation method

\subsection{Flowchart of proposed method}

The overall process of the proposed method can be represented in the flow chart as shown in Fig. 1. First, state equation eqn. 9 is used to obtain the estimated stator currents and rotor flux. Secondly, stator currents are measured. Then the second-order Kalman filter eqns. $14-18$ is utilised to modify the estimated rotor flux based on the measured stator currents. Finally, eqn. 8 is used to obtain the estimated rotor speed. The estimated rotor speed is updated and the whole process repeats again.

\section{Comparison of computation time}

To compare the computation time of various methods we give a table which shows the required simulation time of each method for a sensorless vector control system to run for 10 seconds and simulation time is 10 us. There are in total one million iterations. The simulation is performed on a Pentium $150 \mathrm{MHz}$ PC. Note that the computation time shown in Table 1 includes the calculation of the induction motor model. From Table 1, it is shown that the proposed method can greatly reduce the computation time compared with reduced-order extended Kalman filter and full-order extended Kalman filter. As shown in the experimental results, the proposed method performs much better than the conventional adaptive observer, especially under heavy load conditions.

Table 1: Comparison of computation time for various methods

\begin{tabular}{ll}
\hline Conventional adaptive observer & $270.66 \mathrm{~s}$ \\
Proposed method & $244.40 \mathrm{~s}$ \\
Reduced-order extended Kalman filter & $284.07 \mathrm{~s}$ \\
Full-order extended Kalman filter & $587.86 \mathrm{~s}$ \\
\hline
\end{tabular}


Table 2: Parameters of induction motor

\begin{tabular}{llll}
\hline$R_{S}$ & $2.7 \Omega$ & $R_{r}$ & $2.22 \Omega$ \\
$L_{s}$ & $0.352 \mathrm{H}$ & $L_{r}$ & $0.352 \mathrm{H}$ \\
$L_{m}$ & $0.342 \mathrm{H}$ & $J_{m}$ & $0.0825 \mathrm{~kg} \cdot \mathrm{m}^{2}$ \\
\hline
\end{tabular}

\section{Experimental implementation}

\subsection{Experimental setup}

The block diagram of the speed-sensorless space vector control system with the proposed speed estimation scheme is shown in Fig. 2. The software part is implemented in a Pentium $120 \mathrm{MHz}$ PC. The machine under study is a 3-HP, 4-pole squirrel-cage induction motor, whose parameters are shown in Table 2 . The speed estimation scheme replaces the speed signal feedback path of the conventional vector control. The stator currents are measured with Hall-effect sensors and sampled through an $\mathrm{A} / \mathrm{D}$ card whose sampling rate is $100 \mathrm{kHz}$. The terminal voltages are calculated by the following equations:

$$
\begin{gathered}
v_{d s}=\frac{V_{d c}}{\sqrt{3}}\left(S_{C}-S_{B}\right) \\
v_{q s}=\frac{V_{d c}}{3}\left(2 S_{A}-S_{B}-S_{C}\right)
\end{gathered}
$$

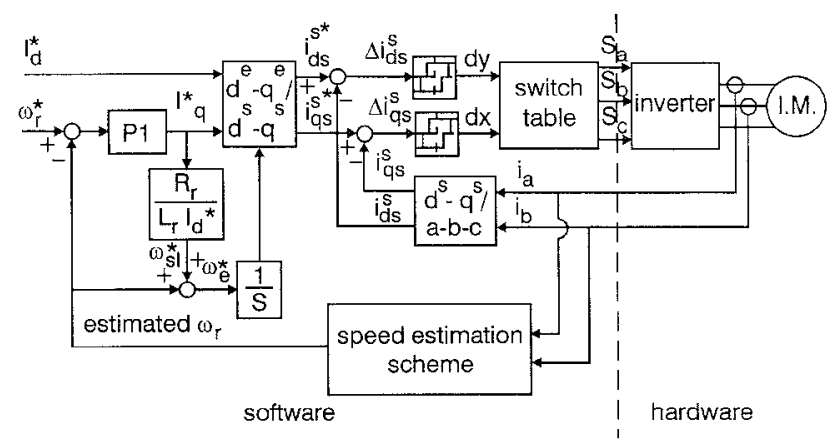

Fig.2 Speed-sensorless space vector control system with new speed estimation scheme

where $S_{A}, S_{B}$ and $S_{C}$ represent the switching states, which can be 1 or 0 , and $V_{d c}$ represents the $\mathrm{DC}$ bus voltage of the inverter, which is assumed constant. One cycle of the control program takes about $49 \mu$ s for the adaptive observer and $84 \mu$ s for the proposed method, i.e. the inverters are switched at about 20 and $12 \mathrm{kHz}$, respectively.

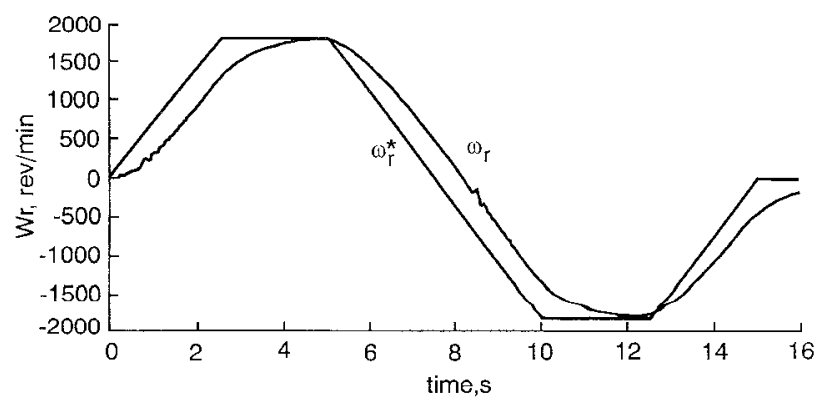

Fig.3 Experimental results of forward-reverse operation under no-load condition using new speed estimation method

\subsection{Experimental results}

Figs. 3 and 4 show experimental results of forwardreverse operation under no-load and full-load conditions, respectively. Fig. 5 shows the experimental results of the steady-state rotor speed under various load conditions using the new speed estimation method. For comparison, Fig. 6 shows the experimental results of the same conditions using the conventional adaptive observer. From Figs. 5 and 6, the new speed estimation method performs better than the conventional adaptive observer, especially under heavy loads.

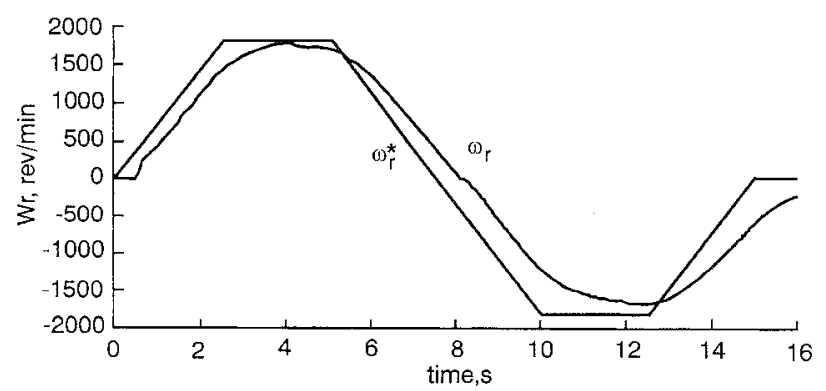

Fig.4 Experimental results of forward-reverse operation under full-load condition using new speed estimation method

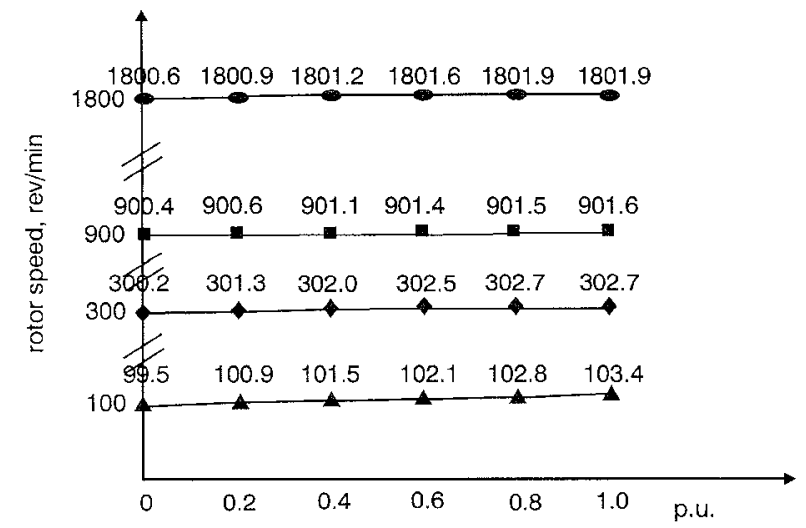

Fig.5 Experimental results of steady-state rotor speed under various load conditions using new speed estimation method

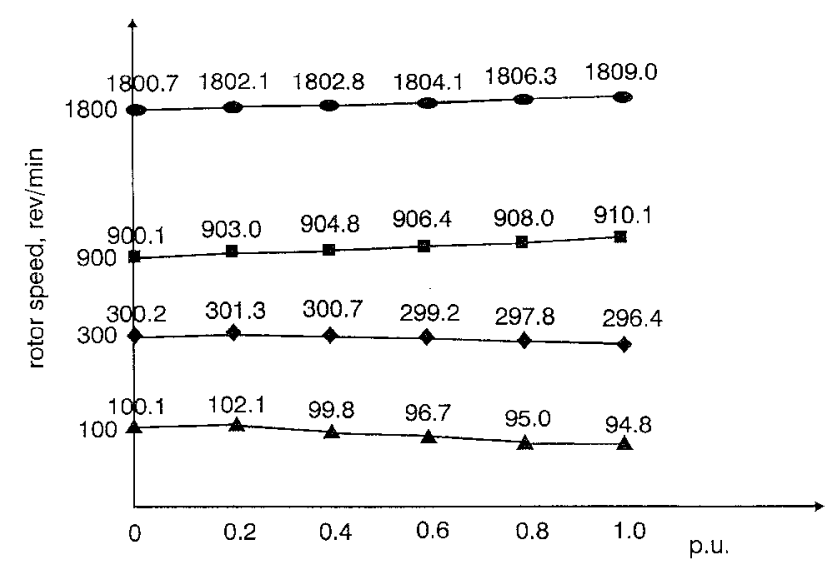

Fig.6 Experimental results of steady-state rotor speed under yarious load conditions using conventional adaptive observer

\section{Conclusions}

We have presented a new speed estimation method of an induction motor based on an adaptive observer in the rotor-speed reference frame in which a secondorder Kalman filter was employed to modify the estimated rotor flux. The new speed estimation method was implemented in a speed-sensorless space vector control system. Experimental results showed that the speed-control performance is good enough that the proposed method could be used in most nonservo applications. Experimental comparison under various 
load conditions between the conventional adaptive observer and the new speed estimation method was also made. The new speed estimation method performed better than the conventional adaptive observer, especially under heavy loads.

\section{References}

1 GABRIEL, R., LEONHARD, W., and NORDBY, C.J.: 'Fieldoriented control of a standard AC motor using microprocessors', IEEE Trans., 1980, IA-16, (2), pp. 186-192

2 KUME, T., and IWAKANE, T.: 'High-performance vector-controlled AC motor drives: applications and new technologies', IEEE Trans., 1987, IA-23, (5), pp. 872-880

3 OGASAWARA, S., AKAGI, H., and NABAE, A.: 'The generalized theory of indirect vector control for AC machines', IEEE Trans., 1988, IA-24, (3), pp. 470-478

4 NILSEN, R., and KAZMIERKOWSKI, M.P.: 'Reduced-order observer with parameter adaption for fast rotor flux estimation in induction machines', IEE Proc. D, 1989, CTA-136, (1), pp. 35-43
5 WIJESUNDERA, D.S., and JACKSON, R.D.: 'Observers for field-oriented control of induction motor drives', IEE Proc. $B$, 1992, EPA-139, (4), pp. 381-386

6 KAO, Y.T., and LIU, C.H.: 'Analysis and design of microprocessor-based vector-controlled induction motor drives', IEEE Trans., 1992, IE-39, (1), pp. 46-54

7 KUBOTA, H., MATSUSE, K., and NAKANO, T.: 'DSP-based speed adaptive flux observer of induction motor', IEEE Trans., 1993, IA-29, (2), pp. 344-348

8 KIM, Y.R., SUL, S.K., and PARK, M.H.: 'Speed sensorless vector control of induction motor using extended Kalman filter', IEEE Trans., 1994, IA-30, (5), pp. 1225-1233

9 KIM, Y.S., KIM, S.U., and YANG, I.W.: 'Implementation of a speed sensorless vector control of induction motor by reducedorder extended Kalman filter'. Proceedings of IEEE-IAS annual meeting, 1995, pp. 197-203

10 TAN, J, and KYRIAKOPOULOS, N.: 'Implementation of a tracking Kalman filter on a digital signal processor', IEEE Trans., 1988, IE-35, (1), pp. 126-134

11 GELB, A.: 'Applied optimal estimation' (Cambridge, MA, MIT Press, 1974) 